\title{
Navigating Tensions About Resident Sexual Expression in Alberta's Continuing Care Homes: a Qualitative Study of Leaders' Experiences
}

\author{
Lisa Howard ${ }^{1}$ (D) Julia Brassolotto ${ }^{1} \cdot$ Alessandro Manduca-Barone $^{1}$ \\ Published online: 13 December 2019 \\ (C) The Author(s) 2019
}

\begin{abstract}
Introduction In continuing care homes, resident sexual expression is complicated by interpersonal, physical, and social features that are part of congregate living. Little is known about how managers navigate the complexities of these features.

Methods We undertook a descriptive, exploratory study to pursue this line of inquiry. We conducted in-depth interviews with 28 participants from a network of managers, clinical ethicists, geriatric specialists, and social workers in Alberta, Canada.

Results We present our findings as three dynamic tensions: 1) the tension between how sexual expression should be supported and how it is addressed in care homes; 2) the tension between private and public space in the care home; and 3) the tension between a medical model of care and a conceptualization of sexual expression as an activity of daily living.

Conclusions These tensions are connected with sexual rights - specifically, with the rights to equality and non-discrimination, privacy, and autonomy and bodily integrity. Sexual expression can also have physical, social, and emotional benefits for continuing care residents. Thus, the tensions surrounding resident sexual expression ought to be navigated with an approach that acknowledges sexual rights and best upholds them.

Policy Implications We suggest that in order to realize the benefits and prevent the harms associated with resident sexual expression, there is both need and opportunity for a coordinated, multi-level approach.
\end{abstract}

Keywords Sexual expression $\cdot$ Long-term care $\cdot$ Qualitative methods $\cdot$ Sexual rights $\cdot$ Managers $\cdot$ Care workers $\cdot$ Residents

Highlights

- Care home managers navigate three tensions related to resident sexual expression

- These tensions are linked to sexual rights of equality, privacy, and autonomy

- A multi-level approach can realize benefits and prevent harms to residents and staff

Electronic supplementary material The online version of this article (https://doi.org/10.1007/s13178-019-00421-0) contains supplementary material, which is available to authorized users.

Lisa Howard

lisa.howard@uleth.ca

Julia Brassolotto

julia.brassolotto@uleth.ca

Alessandro Manduca-Barone

alessandro.manducaba@uleth.ca

1 Faculty of Health Sciences, University of Lethbridge, 4401 University Drive, Lethbridge, AB T1K 3M4, Canada

\section{Introduction and Background}

There is a growing demand for continuing care services for people who, either as a consequence of chronic health challenges, disability, or age-related decline, require assistance with the tasks of daily life (Lafortune \& Balestat, 2007). In the face of accumulated losses associated with moving into continuing care, sexual expression can take on an important meaning for residents (Doll, 2013). Safe and healthy expression of sexuality is recognized as a right (World Association for Sexual Health (WAS), 2015) and a condition of human flourishing across the lifespan (Barrett \& Hinchliff, 2018). By 'sexual expression', we refer to a broad range of identities, practices, acts, and relationships. This can include thoughts and fantasies, sexual orientation, grooming and self-presentation, romantic relationships, handholding, kissing, hugging, bed-sharing, masturbation, and engaging in physical sex acts. There is ample evidence of continuing care residents' ongoing interest in sexual expression and its benefits for their wellbeing (Doll, 2013; Lottmann \& Kollak, 2017; Makimoto, 
Kang, Yamakawa, \& Konno, 2015; McAuliffe, Bauer, Fetherstonhaugh, \& Chenco, 2015).

Despite its benefits, supporting sexual expression in care homes can be complex. Scholars have noted that sexual expression in continuing care is often influenced by a multitude of factors, including the perception that residents are asexual, stigmatization of some forms of sexuality, and organization of living spaces and care practices that displace opportunities for intimacy (Doll, 2013; McAuliffe et al., 2015; Roelofs, Luijkx, \& Embregts, 2015). Supporting this private aspect of a resident's life is further complicated by the many parties who occupy the resident's space for the purposes of providing personal care, observation, and socialization. Residents with diminished cognitive capacity often have agents who legally act as substitute decision-makers and this can further complicate matters of resident sexual expression (Brassolotto, Howard, \& Manduca-Barone, 2019).

\section{Challenges with Sexual Expression in Continuing Care Homes}

The right to sexual expression is often undermined in continuing care homes, which have historically been prohibitive in their responses to matters of resident sexual health. There is often a lack of staff education about sexual expression across the lifespan and an absence of related policies (Bauer, Nay, \& McAuliffe, 2009; Tarzia, Fetherstonhaugh, \& Bauer, 2012; Villar, Fabà, Serrat, \& Celdrán, 2015). The personal perspectives of staff and family members often create limitations for resident sexual expression (Frankowski \& Clark, 2009; Makimoto et al., 2015; McAuliffe et al., 2015; Roelofs et al., 2015). Furthermore, when ageism, sexism, and ableism converge at the topic of sexual health, older persons experience disproportionate amounts of discrimination or stigma from care staff and family members (Doll, 2013; Frankowski \& Clark, 2009). The marginalization is amplified for those who challenge sex and gender norms such as LGBTQ2S+ (lesbian, gay, bisexual, queer, transgender, two-spirited and other) persons (Furlotte, Gladstone, Cosby, \& Fitzgerald, 2016; Lottmann \& Kollak, 2017).

There is a particularly restrictive approach in cases of residents with dementia because staff and family are rightly worried that sexual behavior may be non-consensual or abusive (Di Napoli, Breland, \& Allen, 2013; Roelofs et al., 2015; Villar, Celdrán, Fabà, \& Serrat, 2014). While these are very legitimate concerns, there are approaches available to ensure that persons with dementia engage in safe, consensual, and nourishing sexual activity (Tarzia et al., 2012; Victor \& Guidry-Grimes, 2018; Kontos, Grigorovich, Kontos, \& Miller, 2016). We suggest that, rather than the traditionally restrictive approach that favors the views of staff and family members, a more nuanced conversation that prioritizes consideration of residents' rights, along with those of staff, family, and other residents, is needed. To this end, we applied a sexual rights framework (see supplemental material: Declaration of sexual rights) to resident sexual expression.

\section{A Sexual Rights Framework}

In recognition of the inter-relationship between sexual expression and well-being, the World Association for Sexual Health (WAS) developed an international Declaration of Sexual Rights (Kismödi, Corona, Maticka-Tyndale, Rubio-Aurioles, \& Coleman, 2017; WAS, 2015). The WAS Declaration built upon prior work done in sexual and reproductive justice, and linked sexual rights with human rights already entrenched in national laws. This was a significant step to protecting rights and freedoms considered fundamental to sexual expression. Since its inception, the Declaration is used to inform how governments and professional organizations address family planning, approach gendered discrimination, and counter sexual violence (WAS, 2015). Barrett and Hinchliff (2018) reconceptualized the WAS Declaration to reflect the needs of older persons. They recommended that these revisions serve as a right-based framework to guide policy, education, and research connected with older persons' sexual health. We apply this framework in the context of continuing care.

The application of a sexual rights framework is particularly relevant for this research because older adults currently living in continuing care were born into, or came of age during, the civil rights and feminist movements, as well as the sexual revolution. They have also lived through the legalization of same sex marriage and changing public discourse around sexuality and sexual expression. These historic events in North America drew attention to the taboos, stigma, discrimination, and human rights violations related to sexual health, and recognized human rights related to sexuality (Kismödi et al., 2017; WAS, 2015). Relative to earlier generations, current and upcoming continuing care populations may have broader views about sexuality and different expectations about sexual rights.

\section{Rights Related to Continuing Care Resident Sexual Expression}

Our study highlights an opportunity to specifically foreground the rights to (1) equality/non-discrimination, (2) privacy, and (3) autonomy and bodily integrity. Each of these three wellestablished human rights is associated with a person's sexual expression. First, older people have a right to sexual expression that is free from discrimination based on sexual orientation, age, and/or ability (Barrett \& Hinchliff, 2018). The recognition of this right to equality and non-discrimination is foundational to creating a culture in which healthy sexual expression is normalized and residents are treated with respect and compassion. Second, any existing right to privacy also extends into the realm of sexual life. This includes discretion 
in decision-making about sexual expression and acting upon those decisions without arbitrary interferences or interruption (Barrett \& Hinchliff, 2018). The right to privacy is central for residents to be able to actualize their sexual rights. Third, older people have the right to autonomy and bodily integrity. Bodily integrity refers to the inviolability and right to selfdetermination over one's body. This right thus supports residents' agency to participate in and/or abstain from sexual expression.

\section{The Alberta Context}

In Canada, continuing care service provision is organized and regulated at the provincial level. However, little is known about resident sexual expression in the province of Alberta. Continuing care services in Alberta include home care, supportive living (SL), and long-term care (LTC). For the purposes of this paper, we will discuss SL and LTC. A SL care home provides different levels of support for residents with varying needs and levels of independence. A LTC home provides 24-h nursing and care for residents with complex and unscheduled needs. In Alberta, there are provincial standards to uphold resident rights to privacy, and awareness of such rights are supported through staff training (Alberta Health Continuing Care Branch, 2018; Alberta Seniors \& Community Supports, 2010). However, the care home environment can create ambiguous boundaries for residents' privacy and limit the ability to operationalize these standards. Additionally, with a focus on a medical model of care, resident sexual expression can be treated as a disruption to be managed, rather than a right to be exercised (Rowntree \& Zufferey, 2015). If care staff deem sexual expression inconsequential, problematic, or untimely (Barrett, 2011), then sexual expression goes unrecognized as an activity of daily living.

The national and international research on this topic provides theoretical insight, and local research — such as this project - contributes important empirical data for the province's continuing care sector. In this paper, we outline findings from a study on how managers and those with whom they consult navigate resident sexual expression in continuing care homes in Alberta, Canada.

\section{Methods and Procedures}

In Alberta, there are no provincial policies or formal guidelines for navigating sexual expression in continuing care. Therefore, we undertook a descriptive, exploratory study to address the question of how managers, and those whom they consult, navigate resident sexual expression in continuing care homes. In such a case where little is known about a phenomenon (Hesse-Biber \& Leavy, 2011), an exploratory research design is well suited to draw out participants' experiences. We adhered to the procedures outlined in the Tri-council Policy Statement: Ethical Conduct for Research Involving Humans throughout the course of the research study. The research protocol and interview guides received ethical approval from our university's Research Ethics Board. We also received operational approvals from participating health service agencies. This included the provincial health service agency, two of its subsidiaries, and one faith-based organization.

\section{Participant Recruitment}

We recruited research participants from a province-wide network of continuing care managers and professionals with whom they consult. Managers of care homes are central in sustaining organizational culture and daily operations. In their role, managers engage with resident sexual expression at the nexus of care provision, human resources, and resident/staff/ family relations. For support with decision-making around a sensitive topic, managers often turn to other professionals such as social workers, clinical ethicists, geriatric specialists, and recreation therapists. These parties play an important consultative role in communicating expectations about staff's response to resident sexual expression (Bauer, Haesler, \& Fetherstonhaugh, 2018) and setting the culture of a care home. Additional inclusion criteria required participants to be at least 2 years in their practice area to ensure familiarity with both the context and processes of resident sexual expression. We aimed to recruit 20-25 participants and ended up with 28 participants. Eighteen participants were managers and 10 were advisors. The advisors came from a variety of professional backgrounds, including social work, recreation therapy, clinical ethics, and specialty geriatrics.

We recruited potential participants through research presentations given by the primary and secondary authors at regularly scheduled virtual meetings for managers of care homes and those who provide consultation/support. These meetings brought together leaders from the continuing care sector in Alberta to discuss shared issues and thus provided us with the broadest network possible for participant recruitment. Following the meetings, a third-party administrative support person sent an e-mail invitation to potential participants. People who were interested in the project contacted the investigators directly, by e-mail or telephone. Either the primary or secondary author described the research project, discussed the study activities, and answered prospective participants' questions. Each participant reviewed and signed a consent form prior to the interviews and the investigators informed them of how they could withdraw or modify participation.

\section{Data Collection}

We collected data using semi-structured, open-ended interviews. The primary and/or secondary author led interviews 
at times and locations of convenience for participants. We provided participants in our geographical region the option to participate in person or over the telephone. Participants from elsewhere in the province took part via telephone. We also provided the option of having focus group interviews (with 2-4 participants) in order to accommodate participants' professional and scheduling preferences. The focus group interviews had the same interview guide as the one-to-one interviews. All interviews were semi-structured and guided by questions that drew out (1) participants' descriptions of sexual expression, (2) their perceptions about the place sexual expression has in continuing care, (3) whether there were policies and/or supports related to the topic, (4) examples of related challenges and successful practices, and (5) areas where supports might be needed. Participants were invited to share illustrative examples from their practice. We also had participants work through vignettes that explored sexual expression in continuing care. Vignettes are a recognized strategy to help participants recall and/or consider real life events by projecting themselves into a hypothetical scenario and explore the responses to the scenario more deeply (Törrönen, 2018). The vignettes incorporated topics of privacy for spousal intimacy, same sex relationships, impact of resident sexual expression on care staff, and sexual expression for residents with dementia.

We collected data from December 2017 to June 2018 and ended up with 18 individual interviews and four focus group interviews (28 participants in total). All participants agreed to have the interviews audio recorded using a digital recording device. The interviews lasted between 45 and $75 \mathrm{~min}$ and were transcribed verbatim, assessed for accuracy, stripped of identifiers, and annotated with observations made from the interviews.

\section{Data Analysis}

Consistent with an inductive approach to qualitative research, we concurrently collected and analyzed the data (Braun \& Clarke, 2006). The authors and research associates independently reviewed interview data line by line to identify consistent words, phrases, and accounts. These were organized into categories and formatted into tables. The research team reevaluated (discussed, reviewed, refined) the categories as the data collection progressed to assess the data fit within the categories, the overall comprehensiveness of the categories, and the developing themes. We noted consistent similarities between the one-to-one and focus group interviews and there were no noted differences. As we developed themes, we tested them through concurrent member-checking with several participants. We continued the process of concurrent data collection and analyses until the categories were rich in detail, replicated in several cases, and uncovered no new information.
We then shared a summary of our analysis with four participants in senior roles, and there were no additional contributions or revisions to the analysis. We present the themes in the form of tensions and our discussion situates them within Barrett and Hinchliff's (2018) framework for sexual rights.

\section{Findings}

Our findings reveal the dynamic tensions that our participants navigate related to resident sexual expression. Participants were in agreement that sexual expression was particularly complex to navigate when a resident had diminished capacity due to dementia. We heard descriptions of intense deliberations about capacity and consent. We explore this matter in greater depth in another manuscript (Brassolotto et al., 2019). In this paper, we focus on three key tensions: (1) tension between how sexual expression should be supported and how it is currently addressed in continuing care, (2) tension between private and public space in care homes, and (3) tension between a medical model of resident care and a conceptualization of sexual expression as an activity of daily living.

\section{The Tension Between How Sexual Expression Should Be Supported and How It Is Currently Addressed in the Care Home}

Participants noted that sexual expression encompassed a spectrum of identities, practices, acts, and relationships. Many of the participants indicated that sexual expression is integral to resident life.

"It's just part and parcel of who we are as people. I think it should have the same place as getting up and washing and dressing and eating your meal; it provides a deeper meaning to life" [Participant 8].

"It's not only sexual activity, independent or with a partner. It's the way you present yourself to the world. How you do your hair, your makeup, your nails, the shows you go to, the activities that you participate in, the books you read. It's a form of attachment that provides security and comfort, and it should be a basic need and basic right in care facilities. [Participant 21].

These descriptions reflect how resident sexual expression includes physical intimacy as well as activities embedded in personal care and leisure practices. Our participants indicated that sexual expression is both a human right and a basic need and it should have a place in continuing care. At the same time, they acknowledged that resident sexual expression is fraught, particularly for residents with cognitive impairment, and the ideal of what it could look like was different from their experiences in practice. 


\section{How Sexual Expression Should Be Supported}

Many participants remarked on the importance of sexual expression for both mental and physical well-being. When asked about the place of sexual expression in care homes, there was a consistent message that staff education about resident sexuality should be accompanied by an organizational policy. Only one participant's employer had a policy that addressed sexual expression and guided staff responses. The policy was supplemented by staff education, and the manager's role was to contextualize the policy in practice.

We have a formal policy on privacy, intimacy and sexuality. We offer education, and we have conversations with the team as part of supporting our staff to support the residents. I'm thinking specifically of a situation where we had a resident in one area who was involved with a resident in another part of the building. We made sure to give them privacy, but we were monitoring as well to make sure it was all appropriate [Participant 11].

Such a policy establishes the place of sexual expression in the care home and creates an expectation for staff to honor peoples' rights to non-discrimination, bodily autonomy, and healthy sexual expression (Barrett \& Hinchliff, 2018; Lester, Kohen, Stefanacci, \& Feuerman, 2016; Rowntree \& Zufferey, 2015). In settings where there was no policy, participants indicated that one would be welcomed.

"I think the provincial health authorities could give us some guiding principles that everyone - even faith-based providers or private providers - would be accountable to. There should be some standards that are set as to, if you are a long-term care provider, this is a fundamental aspect of care, and you need to be able to address it in a compassionate and a forthright manner" [Participant 27].

Some participants called attention to sexual orientation as a human right in general, and made particular reference to the needs of LGBTQ2S+ residents. There was agreement that continuing care homes should be inclusive spaces for persons from LGBTQ2S+ communities. Furthermore, "the incoming cohorts will have lived through the sexual revolution and same sex marriage. So [people] coming into care homes will be much more open, much more vocal" [Participant 22]. Several participants saw a role for guidelines on how to create a safe and welcoming environment for residents of all orientations. These guidelines would reinforce education and help to establish consistency of responses within and between care homes. We heard that a policy on sexual expression would also provide clarity for residents about expectations and supports with this part of their care.
In sum, we consistently heard that sexual expression ought to have a place in continuing care homes. Our participants claimed that residents should be supported by staff who have education on this topic, a site-level policy, and sector-level guidelines. Participants were clear that residents should not be stigmatized for expressing their sexuality or discriminated against for their orientation. Despite these clear accounts of how sexual expression should be supported in continuing care homes, our participants indicated that this vision did not reflect their current reality.

\section{How Sexual Expression Is Currently Addressed in Continuing Care}

Challenges with sexual expression in continuing care were largely attributed to care staff's attitudes about sexuality and compounded by the absence of guidelines to support any forms of sexual expression. One participant noted, "a lot of the discomfort [about sexual expression] comes from minimally trained care workers from very religious backgrounds with conservative views about sexuality and managers have to work through all of that" [Participant 20]. Other participants also noted that direct care workers came from diverse educational, cultural, social, and religious backgrounds, which contributed to varying degrees of acceptance of, and comfort with, resident sexual expression. None of the participants suggested that staff views should be homogenous. Rather, they indicated that staff's personal views and preferences should not infringe on resident rights.

Participants noted that resident sexual behavior is often considered problematic and rarely viewed as an attempt by the resident to experience social and/or bodily connection. For residents with dementia, participants had to contend with care staff's interpretation of sexually expressive behaviors as a consequence of 'the disease' rather than a legitimate outlet for connection or pleasure. Given the imperative to protect these residents from harm and mitigate staff concerns about legal reprisal, participants were under pressure to make decisions that limited resident sexual autonomy, "[i]f an individual has a mild or even moderate cognitive impairment, the response from the clinical team has been profoundly conservative when it comes to sexual behavior" [Participant 27]. Sexuality can be a taboo topic in general, and resident sexual expression is particularly replete with difficulties related to staff's assumption that residents will be asexual.

There is the idea among staff that when people come into care the need for sexual expression is gone. So, it's seen as abnormal. To be resident-centered, we have to realise sexual expression is natural and not a 'taboo' subject [Participant 14].

The challenge is we haven't found ways to comfortably acknowledge that sexuality has a place in the care home. 
We talk with staff about this as a healthy part of being human and we shouldn't expect our residents to hide this because they are human beings who have some kind of sexuality and a sexual identity [Participant 13].

However, the talk about normalizing resident sexual expression does not necessarily translate into action. The attitudes of staff members about resident sexuality appear to undermine its place in the care home and temper managers' efforts to support its safe expression. All participants acknowledged that without education and guidelines, the staff relied upon personal values when they encountered instances of resident sexual expression.

I hear comments that the staff find it really distasteful. They don't understand it's an important part of wellbeing and I think it's because there is no education on this. I looked through their curriculum [for Health Care Aides] and this is missing [Participant 23].

I notice the staff bring their own personal biases about what is right and wrong, which really influences how they manage the behaviours. When resident sexual expression comes to my attention, staff see it as a misbehavior [Participant 28].

The staff's personal views can influence their professional work and, in so doing, unjustly constrain residents' rights to sexual expression. We heard that support for same-sex relationships in continuing care is also a 'work in progress'. "A lot of LGBTQ2S+ people are fearful of being open in the longterm care setting. And a lot of elders have experienced extraordinary amounts of discrimination... there is a lot of fear when they disclose" [Participant 27]. A few participants spoke about some promising developments, but we also heard that many educational or supportive efforts are undertaken discreetly to avoid controversy. When these matters are not discussed openly, residents' right to non-discrimination may be infringed upon:

We do have couples' suites. So again, we're just beginning the conversations of what defines a couple. Is it a heterosexual couple? Or is it a same-sex couple as well? I don't think we're having conversations about that. I don't think we've moved to that direction where we would actually admit that same-sex couple into that particular suite [Participant 28].

The distinction between appropriate and inappropriate expressions of sexuality was described as a matter of perspective. For example, there was widespread agreement that unwanted groping was inappropriate, but there was less agreement about whether or not residents should be permitted to view pornography in their bedrooms. Typically, sexuality is only addressed when family, staff, or other residents considered particular expressions to be inappropriate. Residents are rarely provided with information about appropriate or healthy ways to express themselves in continuing care settings.

In the current environment, there appears to be a lack of consistency in care home practices. This contributes to substantially different resident experiences, and creates unjustly arbitrary approaches to resident sexual expression dependent upon where the resident lives and who is on shift. As one participant stated, "we're really just winging it" [Participant 13]. It is important to note that care staff attitudes are not the sole source of challenges. When staff lack education and resources, they improvise to the best of their abilities. A difficulty with "winging it" is that it creates inconsistent responses, which are grounded in staff's values rather than residents' rights.

\section{The Tension Between the Care Home as a Private Home Space and a Public Work Space}

Prior to admission to a care home, an individual's intimate life might not garner attention beyond their personal circle. In the care home, many parties are aware of intimate aspects of the resident's life and occupy their space to provide medical treatment and assistance with activities of daily living. Several participants noted that a resident's bedroom should be considered a private space. However, the bedroom does not readily accommodate privacy because it is also the care staff's workspace.

You know, the amount of meaningful privacy that some of our residents have is pretty minimal...what's at play is a difference in how people understand the space. So, do they see their room as essentially their home, like an apartment almost, like a little bachelor suite? Or is it a bedroom in a larger house? Or is it neither of those things - is it actually just a room and a bed in a health care facility? [Participant 13].

The presence of multiple people in the care home, and the nature of their work with residents, creates an ambiguous boundary between private and public space. For example, if a person was in their own home and watched pornography, this could go unnoticed. However, in a congregate setting, the tension between private and public space surfaces because the care work intersects with the resident's sexual expression. One participant provided an illustrative example of this common experience:

[W] had a gentleman that wanted to view pornographic movies. The staff were very upset by that, they would walk in on him and find things that were offensive to them... So we had to get the team together and decide 
how we would go about this, allow him to have his freedom in his home, which was now the facility, and still manage to respect the rights of the other residents and the staff... [Residents] should have a right to sexual expression, but it should not infringe on the rights of others who are caring for them. So in doing that we need to find a balance. What we did was we talked to him and had a contract with him where he was allowed to [watch porn], but he needed to let the staff know in advance that that's what he was doing by pulling his curtains or closing his door so that anybody coming in would know what they might be walking into [Participant 1].

This example highlights the murky boundary because the resident behaves as if the bedroom is a home space, and the staff interprets viewing pornography as an inappropriate behavior in their workplace. The manager who shared this story indicated that care staff need not extinguish the resident's right to expression. They can give residents space to express themselves privately instead.

Other participants indicated that it can be difficult for residents to explore solitary or shared sexual expression away from public view. Many care homes are "cursed with shared rooms" [Participant 14] and many residents' rooms do not have locks on the doors. Furthermore, there were common occurrences where staff, roommates, or visitors walked in on residents at inopportune moments.

We had two women who formed a relationship. They would go into each other's rooms and pull the curtain, sit on the bed, and listen to music. The staff would walk into the room and catch the couple kissing and expressing their sexuality. The staff were shocked. I had to have a conversation with the staff about privacy. So now they notice the two walkers behind the curtain in the room and do not disturb. If staff have to disturb, they knock and say 'can I come in?' It's becoming something that we consciously make an effort to live with because that is the reality [Participant 3].

In a case where a resident wanted to masturbate in their room and needed some privacy, there was the staff just walking in on them without giving any notice. It takes a big culture change for staff to find some boundaries around privacy so they are not entering the room to check on people without knocking, without respect for what someone could be doing [Participant 10].

The tension between private and public space is heightened in rural continuing care homes. "There tends to be more discomfort around this in smaller towns because people talk" [Participant 15]. Participants noted that relationships between community members, longstanding histories among care home residents, and gossiping by visitors complicated these dynamics.

I'm thinking of a scenario where a friend's father is admitted who then exhibits some sexual desires for somebody other than the mother, than his wife, and you knew the kids growing up. How do you talk about this or who does the family consult? [Participant 1].

It's harder in these rural sites. The family can be embarrassed when they go and visit their husband or their wife and find out they've been doing something that was not part of their personality before because everybody they know and associate with may be related to the staff at the facility [Participant 8].

The nature of rural communities, where people are more likely to know one another and have opportunities to share information, make it more difficult to protect resident privacy. This can be a contentious issue for residents because their sexual expression may be judged or stigmatized by community members. Whether in a small town or a large city, participants indicated that it is reasonable to expect that employees and visitors will safeguard resident health information, exercise discretion in conversations about residents' lives, and respect residents' physical privacy. However, in small towns, this can be more difficult to ensure.

The right to privacy of sexual expression is realized through participation in solitary or shared pursuits without intrusion (Barrett \& Hinchliff, 2018). Our participants were unanimous that the ambiguous boundaries between personal and public spaces in continuing care created tension. The demands of health care provision in a 'home-like' setting make it difficult to maintain resident privacy. When the care home space hampers opportunities for privacy, and the place of resident sexuality is in question, residents' rights may be infringed upon. This tension is further complicated by a medical model of care.

\section{The Tension Between a Medical Model of Care and Sexual Expression as an Activity of Daily Living}

Individuals move into continuing care homes for varying degrees of support with their activities of daily living and management of medical conditions. A focus on residents' clinical needs directs the organization of care priorities toward a medical model. Many of our participants observed that a medical model is reinforced through care home routines that include resident assessment, delivery of personal care services, and health management. This model limits the staff's awareness of, and residents' opportunities for, sexual expression.

The things we have to focus on reflect the medical approach, the clinical approach. So where's that balance 
with quality of life, which includes resident sexual expression? It's easier to support the medical approaches to the resident's clinical issues versus the sexual expression piece [Participant 19].

There is a big focus on the personal and medical care needs - we don't discuss sexuality with residents and families, the staff are not prepared for when residents express themselves, and the institution doesn't bring this forward as a part of care planning. Everyone gets surprised by residents' sexual behaviors [Participant 6].

The right to nourishing and safe sexual experiences goes unrecognized if there is no expectation of staff-or capacity within their work flow-to support sexual expression. Participants said that care staff could support residents by turning one's back and drawing the curtain, providing physical inspections during care after intimacy, exercising discretion when witnessing expressions of sexuality, respecting autonomy and privacy, and upholding residents' right to make choices about safe and healthy sexual expression, even when they may not align with one's own preferences. Some participants suggested that institutional ambivalence about sexual expression as a legitimate aspect of resident life influences the extent to which staff are able to support it. The matter is further complicated when residents cannot independently meet their needs for sexual expression (e.g., applying makeup before a visitor arrives, acquiring romantic novels, opening a bottle of lubricant) and the staff do not see accommodating resident sexuality as part of their work.

As a manager, my immediate response is to respect the resident's wishes about sexuality, but I understand where the staff are coming from because they have tasks to do that are time-sensitive and, therefore, they can't offer much support. They know that if tasks are not completed, there are consequence, so they're task-oriented. [Participant 2].

For the direct care staff, they get task-focused and there is no consideration of the resident's sexual expression. And when it does show, the staff panic because they think this isn't something that's in the scope of their job or part of the care plan [Participant 18].

When care is task-centered, sexuality is typically viewed as problematic and residents have no claim to engage in or have support for sexual expression as an activity of daily living. With the predominant focus on health care needs, it is no surprise that care staff rarely recognize or realize opportunities to proactively anticipate resident sexual expression.

Some of the difficulty with resident sexual expression comes from our task-focused care. It is quite a challenge as facilities are so institutional - it's a top-down approach to people. The time's ten o'clock and lights out you know. 'What's John doing in with Mary? We can't have this! We can't let them do that because we have to do this, and this, and this by midnight' [Participant 23].

If we know up front that someone has a tendency to express himself or herself sexually, it would be considered as part of their disease condition and we would manage it accordingly using behavioral, environmental, or chemical restraints [Participant 5].

As Participant 5 noted, even when sexual expression is anticipated, it is often pathologized. For the employer that had a policy and staff who were willing to support sexual expression, resident intimacy was still scheduled around unit routines.

We still have to coordinate things around care. We'll put the 'do not disturb' sign on the door with the understanding that if we have to go in, we'll knock first. We talk about trimming the person's nails and keeping them clean, making sure they have lubricant, and checking them afterwards - a visual inspection - to make sure everything's okay. [Participant 22].

We heard that even when resident sexual expression is supported by a policy, it can be subordinated to the care home routines. Such an orientation can create an environment in which resident pleasure is treated as another scheduled task. Our participants did not suggest that care schedules ought to be organized around individuals' personal sexual practices, rather that some flexibility and compassion can create a more home-like environment. This flexibility provides opportunity for spontaneity, for residents to feel good in and about their bodies, and to experience themselves as whole beings with desires and choices and not solely the recipients of medical care. The rights to bodily autonomy expressed through solitary or shared intimacy, as well as the privacy to engage in such pursuits, may be overlooked because sexuality is unrecognized as an activity of daily living.

It was widely acknowledged by participants that sexual expression is an important resident right, yet it is also an uncomfortable, even taboo, topic for them to broach with direct care staff. Therefore, there is a tension between how resident sexual expression should look and how it does look. In absence of a formal policy to legitimize sexual expression as a right, this aspect of life is circumscribed by staff's attitudes, beliefs, and values. We heard that care staff get training about medical and personal care, but it is not adequate to help anticipate and support resident sexual expression. Furthermore, there are ambiguous boundaries regarding the division of public and private space. Our participants indicated that neither residents nor care staff were clear on where exactly residents were permitted to engage in sexual pursuits. There were scarce 
supports available to residents for safe and healthy sexual expression because such activities stretch the limits of what the current model of task-centered care can accommodate. Resident rights to sexual expression may become a casualty of the uncertainty surrounding its place in the care home, the ambiguity of boundaries that afford residents privacy, and the rigidity imposed by a medical model of care.

\section{Limitations}

Despite our contributions, there are some limitations to this research. Our findings describe the perspectives of 28 participants and may not reflect the views of all leaders in the continuing care sector. The individuals who participated were comfortable discussing this topic and, though heterogeneous in some of their views, were generally supportive of sexual expression in continuing care. We did not have any participants who were especially uncomfortable with the topic or opposed initiatives to address it. Such adverse perspectives are not represented in our data, though they do exist in the province. Our participants also described sexual expression as a 'right' that was inherent to an individual's realization of their sense of self. This perspective may reflect Western ideals that privilege self-determination and are not universal across cultures and jurisdictions.

\section{Discussion}

In the absence of open conversations, education, and policy, our participants spent considerable time navigating the tensions noted in our findings. An understanding of these tensions is important for ensuring that continuing care homes are desirable places to live or work. Our research findings also confirm some of the key themes from scholarship on the topic from other jurisdictions. For instance, the prevalence of ageist, eroto-phobic attitudes in continuing care, the restrictive physical design of the care home, and the predominant focus on medical tasks (Simpson, Brown Wilson, Brown, Dickinson, \& Horne, 2017; Thys et al., 2019). The same applies for members of the LGBTQ2S+ community, who may experience discrimination in care homes (Furlotte et al., 2016; Mahieu, Cavolo, \& Gastmans, 2019). As noted earlier, fluctuating and diminished capacity can further complicate these tensions. The presence of dementia or traumatic brain injury can certainly influence how staff and families respond to cases of sexual expression (Brassolotto et al., 2019). Collectively, these phenomena work against residents' ability to exercise agency for sexual expression.

Our findings also reveal how these tensions connect with sexual rights. Specifically, the right to (1) equality and nondiscrimination, (2) privacy, and (3) autonomy and bodily integrity. Our findings support the growing body of work on the sexual rights of older people. These sexual rights should be used as the foundation for education and policy regarding sexual expression in continuing care. Only then can we ensure consistency in safeguarding the dignity and healthy sexual expression of residents. More specifically, we recommend a coordinated, multi-level response that includes care homes and the broader continuing care sector.

\section{Recommendations}

At the care home level, we recommend that managers facilitate conversations with their staff to discuss personal values, beliefs, and attitudes and reflect on how these may affect their work. Our research findings confirmed the work of other scholars regarding the impact and prevalence of negative perceptions and limited knowledge that direct care providers have about sexual expression in older persons and people with disabilities (Haesler, Bauer, \& Fetherstonhaugh, 2016; Rowntree \& Zufferey, 2015; Villar, Celdrán, Fabà, \& Serrat, 2017). Staff members should receive targeted sexual expression training (Caceres, Travers, Primiano, Luscombe, \& Dorsen, 2019). The aforementioned conversations can provide managers with an opportunity to contextualize education to staff's experiences in the care home.

There is also a need to support residents' right to privacy. A care home's physical and social spaces make it difficult for residents to exercise privacy in their intimate lives (Villar et al., 2014). There is an opportunity for managers to engage purposefully with care staff and reconceptualize how the space is navigated with an aim of ensuring residents' right to privacy. Some examples could be reducing unannounced intrusions into residents' rooms and exercising discretion in discussions about resident care. When building or renovating a care home, it is important that adaptations are made with privacy considerations in mind. These efforts could enhance general quality of resident life (Burack, Weiner, Reinhardt, \& Annunziato, 2012) and better utilize physical space.

We also recommend a continuing care policy on resident sexual expression. A policy, with education to support its application, can establish the place of resident sexual expression, support consistency in the staff's responses, and communicate what residents can expect. As noted elsewhere, in absence of policy about resident rights to sexual expression, the preferences and priorities of staff and family may eclipse those of residents (Bauer et al., 2018). Several scholars have developed guidelines for how to develop such a policy (Everett, Young, Carlson, \& Clements, 2010; Lester et al., 2016). Our findings highlight the need for these guidelines in order to establish a more consistent approach to sexual expression. An effective policy can attenuate perceptions of sexuality as 'taboo' or 'problematic', and establish it as an activity of daily living. 
At the continuing care sector level, our findings demonstrate the need for a service provision standard about resident sexual expression. A sector level standard should stipulate that staff require targeted education about sexual expression, that the care home design and usage ought to support social and physical privacy, and that site level policies shall outline expectations, roles, and responsibilities for how rights to sexual expression are upheld. A coordinated, multi-level approach that contextualizes sexual rights in continuing care could go a long way towards supporting safe, healthy, and dignified sexual expression.

\section{Conclusion}

These recommendations serve as an important starting point to address the tensions described above. With a coordinated approach, we can work towards realizing participants' accounts of how sexual expression in continuing care should be supported, have greater clarity about where residents are able to enjoy privacy, and destigmatize sexual expression as an activity of daily living. These changes at site and sectoral levels will make it easier for managers and care staff to navigate these tensions and to better uphold the sexual rights of continuing care residents. Our findings reveal the breadth and urgency of the work to be done.

Open Access This article is licensed under a Creative Commons Attribution 4.0 International License, which permits use, sharing, adaptation, distribution and reproduction in any medium or format, as long as you give appropriate credit to the original author(s) and the source, provide a link to the Creative Commons licence, and indicate if changes were made. The images or other third party material in this article are included in the article's Creative Commons licence, unless indicated otherwise in a credit line to the material. If material is not included in the article's Creative Commons licence and your intended use is not permitted by statutory regulation or exceeds the permitted use, you will need to obtain permission directly from the copyright holder. To view a copy of this licence, visit http://creativecommons.org/licenses/by/4.0/.

\section{References}

Alberta Health Continuing Care Branch (2018). Continuing care health service standards. Retrieved from: https://open.alberta.ca/ publications/9781460138441. Accessed 14 Feb 2019.

Alberta Seniors and Community Supports (2010). Long-term care accommodation standards. Retrieved from: https://open.alberta.ca/ publications/4840070. Accessed 14 Feb 2019.

Barrett, C. (2011). Auditing organizational capacity to promote the sexual health of older people. Electronic Journal of Applied Psychology, 7(1), 31-36. https://doi.org/10.7790/ejap.v7i1.233.

Barrett, C., \& Hinchliff, S. (Eds.). (2018). Addressing the sexual rights of older people: Theory, policy and practice. New York: Routledge.

Bauer, M., Haesler, E., \& Fetherstonhaugh, D. (2018). Organisational enablers and barriers to the recognition of sexuality in aged care:
A systematic review. Journal of Nursing Management, 27(4), 858868. https://doi.org/10.1111/jonm.12743.

Bauer, M., Nay, R., \& McAuliffe, L. (2009). Catering to love, sex, and intimacy in residential aged care: What information is provided to consumers? Sexuality and Disability, 27(1), 3-9. https://doi.org/10. 1007/s11195-008-9106-8.

Brassolotto, J., Howard, L., \& Manduca-Barone, A. (2019). Sexual expression in Alberta's continuing care homes: Capacity, consent, and co-decision-making. Canadian Journal of Aging.

Braun, V., \& Clarke, V. (2006). Using thematic analysis in psychology. Qualitative Research in Psychology, 3(2), 77-101. https://doi.org/ 10.1191/1478088706qp063oa.

Burack, O., Weiner, A., Reinhardt, J., \& Annunziato, R. (2012). What matters most to nursing home elders: Quality of life in the nursing home. Journal of the American Medical Directors Association, 13(1), 48-53. https://doi.org/10.1016/j.jamda.2010.08.002.

Caceres, B., Travers, J., Primiano, J., Luscombe, R., \& Dorsen, C. (2019). Provider and LGBT individuals' perspectives on LGBT issues in long-term care: A systemic review. The Gerontologist, 1-15. https://doi.org/10.1093/geront/gnz012.

Di Napoli, E., Breland, G., \& Allen, R. (2013). Staff knowledge and perceptions of sexuality and dementia of older adults in nursing homes. Journal of Aging and Health, 25(7), 1087-1105. https:// doi.org/10.1177/0898264313494802.

Doll, G. (2013). Sexuality in nursing homes: Practice and policy. Journal of Gerontological Nursing, 39(7), 30-39. https://doi.org/10.3928/ 00989134-20130418-01.

Everett, B., Young, J., Carlson, M., \& Clements, G. (2010). New British Columbia guidelines for supporting sexual health and intimacy in care facilities. Healthcare Management Forum, 23(1), 21-24. https://doi.org/10.1016/j.hcmf.2010.02.001.

Frankowski, A., \& Clark, L. (2009). Sexuality and intimacy in assisted living: Residents' perspectives and experiences. Sexuality Research and Social Policy Journal of NSRC, 6(4), 25-37. https://doi.org/10. 1525/srsp.2009.6.4.25.

Furlotte, C., Gladstone, J., Cosby, R., \& Fitzgerald, K. (2016). "Could we hold hands?" older lesbian and gay couples'perceptions of longterm care homes and home care. Canadian Journal on Aging / La Revue Canadienne Du Vieillissement, 35(4), 432-446. https://doi. org/10.1017/S0714980816000489.

Haesler, E., Bauer, M., \& Fetherstonhaugh, D. (2016). Sexuality, sexual health and older people: A systematic review of research on the knowledge and attitudes of health professionals. Nurse Education Today, 40, 57-71. https://doi.org/10.1016/j.nedt.2016.02.012.

Hesse-Biber, S., \& Leavy, P. (2011). The practice of qualitative research (2nd ed.). Los Angeles: Sage Publications.

Kismödi, E., Corona, E., Maticka-Tyndale, E., Rubio-Aurioles, E., \& Coleman, E. (2017). Sexual rights as human rights: A guide for the WAS declaration of sexual rights. International Journal of Sexual Health, 29(sup1), 1-92. https://doi.org/10.1080/19317611. 2017.1353865.

Kontos, P., Grigorovich, A., Kontos, A. P., \& Miller, K. (2016). Citizenship, human rights, and dementia: Towards a new embodied relational ethic of sexuality. Dementia, 15(3), 315-329. https://doi. org/10.1177/1471301216636258.

Lafortune, G., \& Balestat, G. (2007). Trends in severe disability among elderly people: Assessing the evidence in 12 OECD countries and the future implications (research report no. 26). Retrieved from OECD publishing website: https://www.oecd-ilibrary.org/socialissues-migration-health/trends-in-severe-disability-among-elderlypeople_217072070078. Accessed 14 Feb 2019.

Lester, P., Kohen, I., Stefanacci, R., \& Feuerman, M. (2016). Sex in nursing homes: A survey of nursing home policies governing resident sexual activity. Journal of the American Medical Directors Association, 17(1), 71-74. https://doi.org/10.1016/j.jamda.2015. 08.013. 
Lottmann, R., \& Kollak, I. (2017). Aging \& diversity: LGBT seniors and long-term care. The Journal of Sexual Medicine, 14, e288-e288. https://doi.org/10.1016/j.jsxm.2017.04.386.

Mahieu, L., Cavolo, C., \& Gastmans, C. (2019). How do communitydwelling LGBT people perceive sexuality in residential aged care? A systematic literature review. Aging \& Mental Health, 23(5), 529540. https://doi.org/10.1080/13607863.2018.1428938.

Makimoto, K., Kang, H. S., Yamakawa, M., \& Konno, R. (2015). An integrated literature review on sexuality of elderly nursing home residents with dementia: Sexuality of elderly with dementia. International Journal of Nursing Practice, 2(Suppl. 2), 80-90. https://doi.org/10.1111/ijn.12317.

McAuliffe, L., Bauer, M., Fetherstonhaugh, D., \& Chenco, C. (2015). Assessment of sexual health and sexual needs in residential aged care: Sexual health and needs in aged care. Australasian Journal on Ageing, 34(3), 183-188. https://doi.org/10.1111/ajag.12181.

Roelofs, T., Luijkx, K., \& Embregts, P. (2015). Intimacy and sexuality of nursing home residents with dementia: A systematic review. International Psychogeriatrics, 27(3), 367-384. https://doi.org/10. 1017/S1041610214002373.

Rowntree, M., \& Zufferey, C. (2015). Need or right: Sexual expression and intimacy in aged care. Journal of Aging Studies, 35(1), 20-25. https://doi.org/10.1016/j.jaging.2015.07.003.

Simpson, P., Brown Wilson, C., Brown, L. J., Dickinson, T., \& Horne, M. (2017). The challenges and opportunities in researching intimacy and sexuality in care homes accommodating older people: A feasibility study, Journal of Advanced Nursing., 73(1), 127-137. https:// doi.org/10.1111/jan.13080.

Tarzia, L., Fetherstonhaugh, D., \& Bauer, M. (2012). Dementia, sexuality and consent in residential aged care facilities. Journal of Medical Ethics, 38(10), 609-613. https://doi.org/10.1136/medethics-2011100453.
Thys, K., Mahieu, L., Cavolo, A., Hensen, C., Dierckx de Casterlé, B., \& Gastmans, C. (2019). Nurses' experiences and reactions towards intimacy and sexuality expressions by nursing home residents: A qualitative study. Journal of Clinical Nursing, 28(5-6), 836-849. https://doi.org/10.1111/jocn.14680.

Törrönen, J. (2018). Using vignettes in qualitative interviews as clues, microcosms or provokers. Qualitative Research Journal, 18(3), 276-286. https://doi.org/10.1108/QRJ-D-17-00055.

Victor, E., \& Guidry-Grimes, L. (2018). Relational autonomy in action: Rethinking dementia and sexuality in care facilities. Nursing Ethics, 1. https://doi.org/10.1177/0969733018780527.

Villar, F., Celdrán, M., Fabà, J., \& Serrat, R. (2014). Barriers to sexual expression in residential aged care facilities: Comparison of staff and residents' views. Journal of Advanced Nursing, 70(11), 2518-2527. https://doi.org/10.1111/jan.12398.

Villar, F., Celdrán, M., Fabà, J., \& Serrat, R. (2017). Staff members' perceived training needs regarding sexuality in residential aged care facilities. Gerontology \& Geriatrics Education, 38(4), 443-452. https://doi.org/10.1080/02701960.2015.1127811.

Villar, F., Fabà, J., Serrat, R., \& Celdrán, M. (2015). What happens in their bedrooms stays in their bedrooms: Staff and residents' reactions toward male-female sexual intercourse in residential aged care facilities. Journal of Sex Research, 52(9), 1054-1063. https://doi. org/10.1080/00224499.2014.959882.

World Association for Sexual Health (WAS). (2015). Declaration of sexual rights. WAS Advisory Council: Author.

Publisher's Note Springer Nature remains neutral with regard to jurisdictional claims in published maps and institutional affiliations. 